\section{ASSOCIATION OF FRAILTY WITH QUALITY OF LIFE IN OCTOGENARIANS WITH SYMPTOMATIC CORONARY ARTERY DISEASE}

${ }^{1}$ Shouaib Qayyum*, ${ }^{2}$ Angela Hoye, ${ }^{3}$ Miriam Johnson, ${ }^{2}$ Richard Oliver, ${ }^{2} \mathrm{~J} J o h n,{ }^{2} \mathrm{D}$ Ngaage, ${ }^{2} \mathrm{M}$ Loubani. ${ }^{1}$ University Hospital North Midlands NHS Trust; ${ }^{2}$ Hull and East Yorkshire NHS Trust:; ${ }^{3}$ Hull York Medical School

\subsection{6/heartjnl-2019-BCS.155}

Background As the population ages, more elderly patients are presenting with symptomatic coronary artery disease (CAD). However, there is lack of evidence regarding the best therapy in this population especially with increasing frailty.

Purpose To determine the relationship between frailty and quality of life (QoL) of octogenarian patients presenting with symptomatic CAD.

Methods We enrolled 150 consecutive unselected patients aged 80 , presenting with acute coronary syndromes and stable angina (SA). Assessments were done at baseline for frailty using Fried frailty phenotype (FFP) and Edmonton frailty scale (EFS). QoL was assessed using Short survey form (SF-12). Comorbidity was quantified using Charlson's comorbidity Index. After the participants had undergone their respective treatments they were reassessed for frailty and quality of life at 4 months.

Results The mean age was $83.7 \pm 3.2$ years. Ninety-nine $(66 \%)$ of the participants were male. Average Charlson's comorbidity index was $6.5 \pm 2.2$. There was significant overlap between frailty comorbidity and frailty $(73.8 \%)$ fig. 2 . Presentation was Stable angina in 68(45.3\%), NSTEMI 45(35\%), STEMI 21 (14\%), and unstable angina 16(10.7\%). Treatment was PCI in 51(34\%), $15(10 \%)$ underwent CABG surgery and $84(56 \%)$ were treated medically. Frailty was present in $26 \%$ and $28 \%$ by EFS and FFP respectively and was more prevalent in females (FFP $37.3 \%$ vs $23.3 \%$ p $=0.07$, EFS $41.2 \%$ vs $18.2 \%$ $\mathrm{p}=0.03)$. Frail participants had higher symptom burden. Regression analysis showed that QoL was significantly related to frailty and Charlson's comorbidity score. FFP was significantly related to the physical composite score for QoL, whilst EFS was significantly related to mental composite score for QoL $(\mathrm{p}=0.003)$. Frail patients with ACS had longer stay in hospital (6.5 vs 2.7 days, $p=0.05)$. At 4 months, 14(9.35) had died, with frail participants having the lowest survival $(50 \%$ vs $26.2 \% \mathrm{p}=0.002)$. Of the remaining 136 participants, $103(75.5 \%)$ were re-assessed. There was a trend towards improvement in symptoms in all treatment arms but made no significant difference to frailty or QoL.

Conclusion In octogenarians presenting with symptomatic CAD, frailty is significantly related to $\mathrm{QoL}$ and is a predictor of survival.

Conflict of Interest None

\section{DSE AUDIT - MATER INFIRMORUM HOSPITAL, BELFAST}

Brian Cox*, Patrick Thompson, Suzanne Maynard. Belfast Trust

\subsection{6/heartjnl-2019-BCS.156}

Introduction Stress echocardiography is a well-established, functional test used in the investigation of chest pain. Its advantages are wide availability, safety and low cost. It forms a part of the ESC guideline for patients with an intermediate probability of stable coronary artery disease. It is a useful test for patients admitted with chest pain and negative cardiac biomarkers prior to discharge.

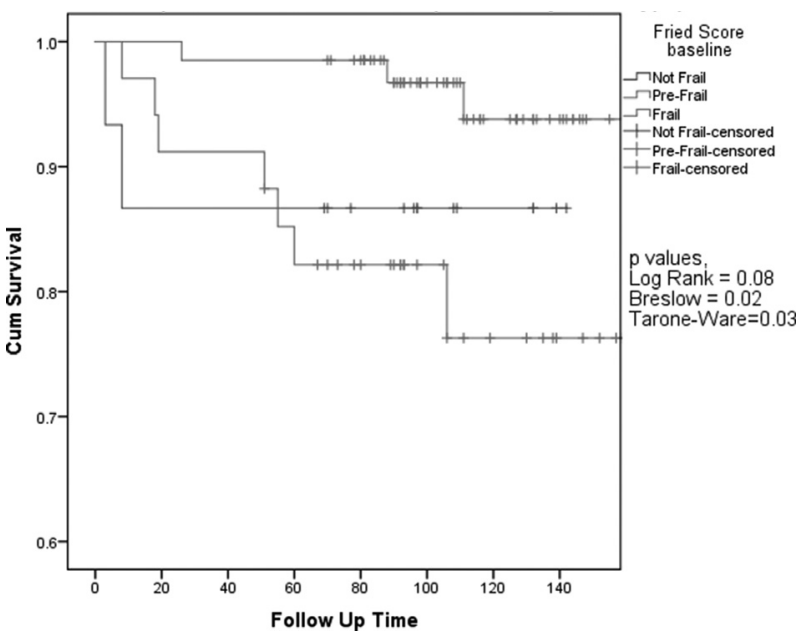

Abstract 158 Figure 1 Kaplan-Meier Survival Curve (Fried Frailty Phenotype)

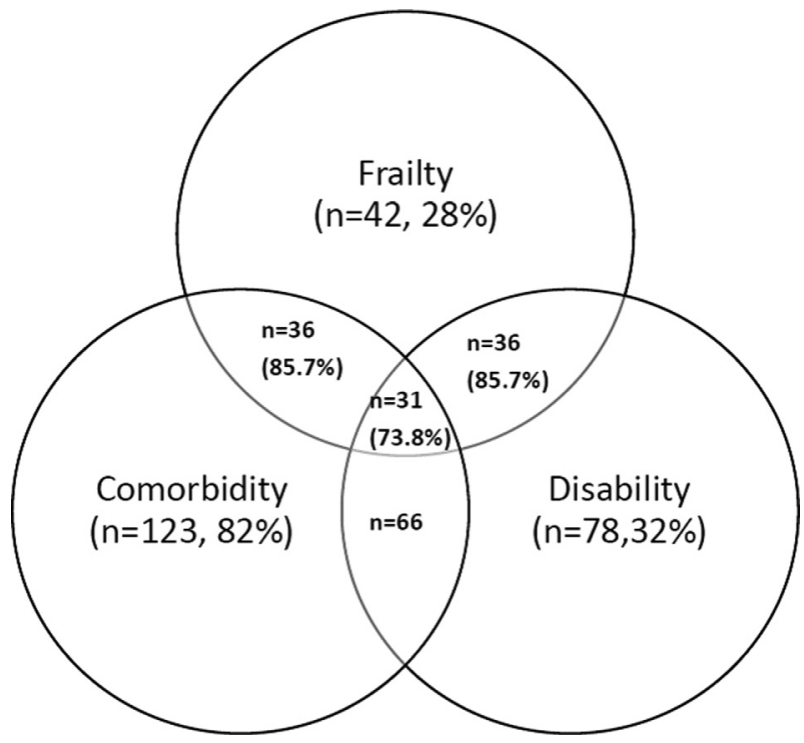

Abstract 158 Figure 2

Methods The goal of this study was to investigate the rate of acute cardiac events within 2 years of a negative DSE.

Data was collected from dobutamine stress echocardiograms (DSE) performed over a continuous 24 month period between February 2014 \& January 2016 in the Mater Infirmorum Hospital (MIH) Cardiac Investigations Laboratory, Belfast.

During DSE, an incremental dobutamine infusion protocol was used to achieve a heart rate of $85 \%$ maximum predicted. A negative result was where no areas of inducible ischaemia were demonstrated in a patient who had met their target heart rate.

The data collection proforma was based upon British Society of Echocardiography recommendations. The patients' Electronic Care Record was used for data collection, which included; demographics, history of ischaemic heart disease, DSE reports, reason for referral and any cardiac events in the following two years. 